\title{
Steering Transforms the Cortical Representation of Self-Movement from Direction to Destination
}

\author{
Michael S. Jacob and Charles J. Duffy \\ Department of Neurology, Neurobiology, and Anatomy, Department of Ophthalmology, Brain, and Cognitive Sciences, and the Center for Visual Science, \\ University of Rochester Medical Center, Rochester, New York 14642
}

Steering demands rapid responses to heading deviations and uses optic flow to redirect self-movement toward the intended destination. We trained monkeys in a naturalistic steering paradigm and recorded dorsal medial superior temporal area (MSTd) cortical neuronal responses to the visual motion and spatial location cues in optic flow. We found that neuronal responses to the initial heading direction are dominated by the optic flow's global radial pattern cue. Responses to subsequently imposed heading deviations are dominated by the local direction of motion cue. Finally, as the monkey steers its heading back to the goal location, responses are dominated by the spatial location cue, the screen location of the flow field's center of motion. We conclude that MSTd responses are not rigidly linked to specific stimuli, but rather are transformed by the task relevance of cues that guide performance in learned, naturalistic behaviors.

Key words: cortical neurons; navigational cognition; optic flow; spatial vision; steering control

\section{Significance Statement}

Unplanned heading changes trigger lifesaving steering back to a goal. Conventionally, such behaviors are thought of as cortical sensory-motor reflex arcs. We find that a more reciprocal process underlies such cycles of perception and action, rapidly transforming visual processing to suit each stage of the task. When monkeys monitor their simulated self-movement, dorsal medial superior temporal area (MSTd) neurons represent their current heading direction. When monkeys steer to recover from an unplanned change in heading direction, MSTd shifts toward representing the goal location. We hypothesize that this transformation reflects the reweighting of bottom-up visual motion signals and top-down spatial location signals, reshaping MSTd's response properties through task-dependent interactions with adjacent cortical areas.

\section{Introduction}

Steering relies on a feedback control loop coupling heading perception with motor control: heading deviations are seen and trigger steering back toward the intended destination (Fig. $1 A$ ). The cortical analysis of optic flow accesses heading cues in its global radial pattern, local motion directions, and the location of its radial center-of-motion (COM) (Gibson, 1950; Warren, 1995).

Optic flow analysis relies on neurons in the dorsal medial superior temporal area (MSTd) (Tanaka and Saito, 1989; Duffy and Wurtz, 1991a,b; Lappe et al., 1996; Britten and Van Wezel,

Received June 21, 2015; revised Sept. 27, 2015; accepted 0ct. 15, 2015.

Author contributions: M.S.J. and C.J.D. designed research; M.S.J. and C.J.D. performed research; M.S.J. and C.J.D. contributed unpublished reagents/analytic tools; M.S.J. and C.J.D. analyzed data; M.S.J. and C.J.D. wrote the paper.

This work was supported by the National Eye Institute (Grant R01-EY022062) and the Office of Naval Research (Grant N000141110525). We thank William Vaughn for the computer programming, systems management, and scientific contributions and Sherry Estes and the URMC DLAM and Vivarium staff for animal care and training.

The authors declare no competing financial interests.

Correspondence should be addressed to Charles J. Duffy, Department of Neurology, University of Rochester Medical Center, 601 Elmwood Avenue, Rochester, NY 14642-0673. E-mail: Charles_Duffy@urmc.rochester.edu.

DOI:10.1523/JNEUROSCI.2368-15.2015

Copyright $\odot 2015$ the authors $\quad 0270-6474 / 15 / 3516055-09 \$ 15.00 / 0$
2002), which is embedded in the dorsal processing pathway (Ungerleider and Mishkin, 1982). MSTd receives bottom-up local motion signals from adjacent middle temporal cortex (MT) (Zeki, 1974) and top-down spatial location signals from adjacent posterior parietal cortex (PPC) (Andersen et al., 1990; Bisley and Goldberg, 2003).

Cueing attention to specific locations enhances the amplitude and selectivity of responses in MT (Seidemann and Newsome, 1999; Treue and Martinez Trujillo, 1999; Wegener et al., 2004) and PPC (Snyder et al., 2000; Saalmann et al., 2007; Herrington and Assad, 2010), so it is no surprise that MSTd's optic flow responses are comparably shaped by attentional cues (Dubin and Duffy, 2007; Sato et al., 2013) but also by tasks that shift attention without explicit cueing of a particular location (Page and Duffy, 2008; Kishore et al., 2012).

We hypothesized that MSTd is contextually adaptive, adjusting the impact of bottom-up and top-down signals to suit the instantaneous demands of learned tasks. To test this hypothesis, we trained monkeys in a steering paradigm that presents optic flow stimuli in a manner that enables our distinguishing neuronal responsiveness to several heading cues embedded in optic flow 
A On-the-Road Lane Keeping
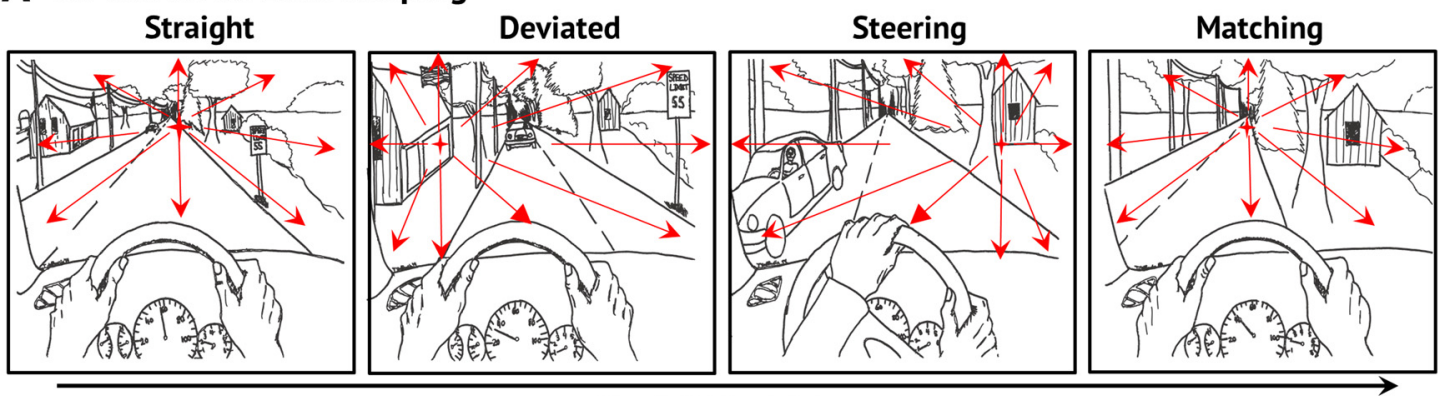

Time in Driving ( $5 \mathrm{~s})$

\section{B Optic Flow Stimulus Set}

Radial Expansion (out)

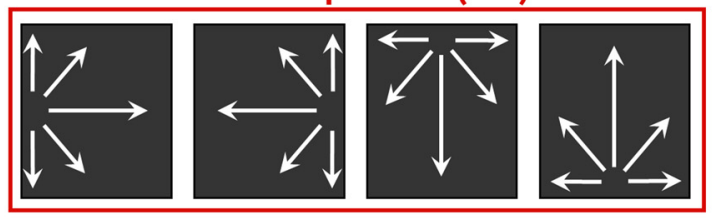

Radial Contraction (in)

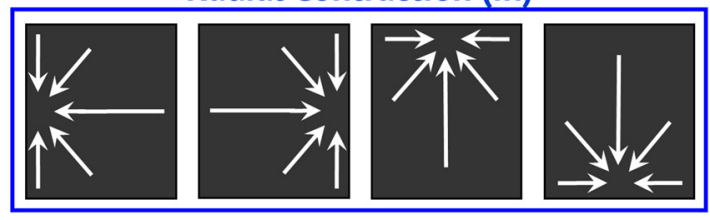

C Delayed Steer to Sample Task
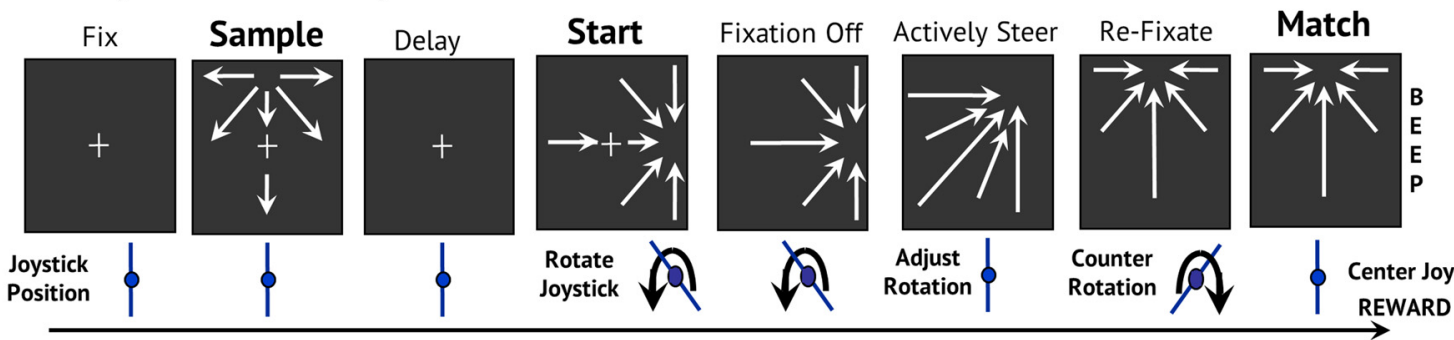

Eye
Position

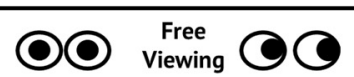

Time in Trial ( $5 \mathrm{~s})$

\section{Joystick and Eye Position}

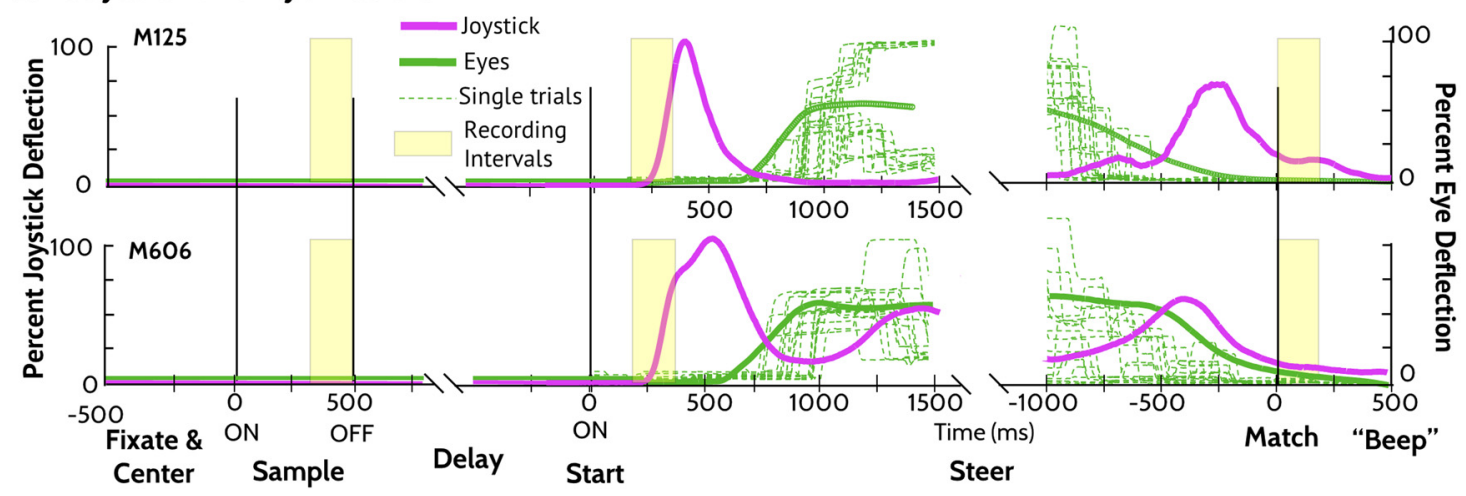

Figure 1. Steering task, stimulus set, behavioral paradigm, and performance. $\boldsymbol{A}$, Steering demands monitoring for unpredictable heading changes and steering to redirect heading back to the intended direction (illustrated from left to right). $\boldsymbol{C}$, In the delayed steer to Sample task, the monkey maintained centered fixation while one of eight Sample optic flow stimuli was presented for $500 \mathrm{~ms}$. After a $1000 \pm 250 \mathrm{~ms}$ blank screen delay, the Start optic flow was presented with a radial COM $90^{\circ} \mathrm{Clockwise} \mathrm{(CW)} \mathrm{or} \mathrm{counterclockwise} \mathrm{(CC)} \mathrm{from} \mathrm{Sample} \mathrm{COM.}$ After 500 ms, free viewing was allowed while the monkey used the rotational joystick to steer the COM to match the sample position, where it was held for 500 ms. When the fixation control was released during active steering, both monkeys saccaded to the COM and then tracked it to the Match location, prompting them to recenter their gaze to earn liquid reward. $\boldsymbol{B}$, The stimulus set consisted of eight optic flow patterns, four outward (left) and four inward (right). D, Both monkeys performed well, as seen in average joystick (magenta, mean deflection from upright) and eye (green, eccentricity from centered fixation) position traces. Example eye traces from a single recording session are shown (dashed, green lines).

(e.g., global radial motion patterns, local motion directions, COM spatial location). We find that steering transforms MSTd's response properties to represent different cues in different stages of the task.

\section{Materials and Methods}

Animal preparation. We studied two adult Rhesus monkeys (Macaca mulatta; M125, female; M606, male) after the surgical preparation of bilateral recording cylinders centered above MSTd (AP: 2 mm, ML: 15 
$\mathrm{mm}$, angle $0^{\circ}$ ) and scleral search coils (Judge et al., 1980). The recording chambers, eye coil connectors, and a head holder were embedded in a dental acrylic cap covering the calvarium. Surgery under general anesthesia used inhaled isoflurane, with banamine $(1 \mathrm{mg} / \mathrm{kg}$, i.m.) postoperative analgesia. All protocols were approved by the Institute Animal Care and the Use Committee and complied with the Public Health Service Policy on the humane care and use of laboratory animals. The monkeys were first trained to maintain ocular fixation while viewing visual motion and then underwent extensive training in the delayed steer to Sample task described below. Monkey M606 received 1 year of training (>1000 h). Monkey M125 was already familiar with joystick steering by optic flow and training occurred over several months $(>200 \mathrm{~h})$.

Optic flow stimuli. Full-field optic flow $\left(90^{\circ} \times 90^{\circ}\right)$ was generated using 680 white dots $\left(0.19^{\circ}\right.$ at $\left.2.61 \mathrm{~cd} / \mathrm{m}^{2}\right)$ on a black background $\left(0.18 \mathrm{~cd} / \mathrm{m}^{2}\right)$. Each dot had a randomly assigned screen duration of 33-1000 ms that maintained uniform dot density across the stimuli. Radial patterns were created by accelerating the dots across screen using a sine $X$ cosine function of each dot's angular distance from the radial COM as viewed by the monkey. Average dot speed was adjusted to $36 \%$ in all stimuli. Both radial in (contraction) and radial out (expansion) were used with four COMs arranged on the cardinal axes at an eccentricity of $24^{\circ}$.

We created a delayed steer to Sample task in which the various cues imbedded in optic might take on more or less relevance in different stages of the task. The Sample condition's random selection of one of four inward or four outward optic flow fields might create a circumstance in which the unbiased representation of flow fields is to be preferred. The shifting of the optic flow field in the Start stimulus by a consistent $\pm 90^{\circ}$ might encourage the monkey to use a local motion direction change detection strategy to guide the better steering wheel response. The consistent goal of returning the COM in the optic flow, whether it is an inward or outward radial pattern, might promote a spatial location based strategy to the monkeys' determining when steering should be adjusted to terminate COM movement at the target spatial location.

Steering paradigm. In our delayed match to Sample paradigm, all optic flow stimuli after the Sample are nonmatches. The task is to rotate their COM locations to match that presented in the Sample (Fig. 1C). At the beginning of each trial, the monkey established centered fixation on a "+" $\left(0.3^{\circ} \times 0.3^{\circ}\right)$ and vertical joystick orientation by rotating it in position alignment mode: a vertical handle aligns fiducial markers on the screen to be just above and below the fixation point. Eye or joystick movements before the steering period aborted the trial.

After correct joystick and eye position were established, the joystick alignment markers were extinguished but the fixation point remained. One of eight Sample optic flow stimuli (four in and four out, cardinal axis positioned COMs) was then presented for $500 \mathrm{~ms}$, followed by a $1000 \mathrm{~ms}$ (plus a random period up to $500 \mathrm{~ms}$ ) fixation only delay period. During the Sample stimulus, 8 stationary dots $\left(0.1^{\circ} \times 0.1^{\circ}\right)$ appeared at the 4 cardinal and 4 oblique locations as landmarks that remained present throughout all trials.

A second optic flow stimulus was then presented with its COM rotated $90^{\circ}$ clockwise or counterclockwise from the Sample's COM. That marked the beginning of the Start period with the joystick switching to steering mode in which its angular deflection specified the COM's angular speed around the circle through all possible COM locations. The monkey was allowed to begin joystick rotation at any time after the appearance of the Start optic flow. However, the monkey was required to maintain centered fixation for the first $500 \mathrm{~ms}$ to ensure stable centered gaze for the analysis of neuronal activity.

While steering the Start COM to match that in the Sample, the fixation point was extinguished and the monkeys were allowed free viewing of the changing optic flow and the eight position markers. Maximum joystick deflection induced a rate of radial center displacement of $125.0^{\circ} \%$. After the monkey brought the COM to within $\pm 5^{\circ}$ of the Sample location for $1000 \mathrm{~ms}$, an audible "beep" was sounded, the optic flow was extinguished, and if the monkey sustained fixation, a liquid reward was delivered. The average steering period was $2-3 \mathrm{~s}$.

We allowed free viewing during steering but required refixation to earn the liquid reward. Both monkeys spontaneously reestablished centered gaze within the previously established fixation windows $\left( \pm 5^{\circ}\right)$ be- fore the beginning of the Match period (mean eccentricity \pm SEM: M125: $\left.0.59^{\circ} \pm 0.01^{\circ}, \mathrm{M} 606: 3.26^{\circ} \pm 0.06^{\circ}\right)$. Despite these small differences in fixation, there was no monkey $X$ stimulus interaction in neuronal cue selectivity.

Behavioral and neuronal data were recorded in 14,214 trials for Monkey M606 and in 6219 trials for Monkey M125. Both monkeys performed the task at $>80 \%$ correct. Fixation errors, initial steering direction errors, and failure to correctly match errors all aborted trials. Behavioral response latency was calculated from the time of Start stimulus onset until joystick rotation.

Recording sites. Single-neuron recordings were directed to MSTd using stereotaxic positioning and confirmed by MR imaging. When experiments were completed, the animals underwent pentobarbital euthanasia and perfusion with heparinized saline followed by formalin. The brain was removed for additional weeks of formalin fixation, followed by blocking and then sectioning at $50 \mu \mathrm{m}$ with every fourth and fifth section stained by the Nissl and Luxol fast blue. Electrolytic lesions and anatomic landmarks were used to localize the recording sites. This analysis indicates that the neurons studied were in the anterior bank of the superior temporal sulcus within the MSTd (Komatsu and Wurtz, 1988).

Single neuron recording. Single-neuron action potentials were recorded using epoxy-coated tungsten microelectrodes (FHC and Microprobe) passed through transdural guide tubes mounted in the recording chambers (Crist et al., 1988). Activity was monitored as the electrode advanced to identify the depth of gray and white matter landmarks. On isolating a neuron, its receptive field was mapped using projected images. MSTd neurons were identified by large receptive fields $\left(>20^{\circ}\right)$ that included the fixation point and showed direction-selective responses preferring large moving patterns (Komatsu and Wurtz, 1988; Duffy and Wurtz, 1991a, 1995). Gray matter layers that included MSTd neurons at appropriate electrode positions were considered to be part of MSTd. Template matching (Alpha Omega) was used to digitize neuronal discharge times that were stored with stimulus and behavioral event markers from the REX system (Hays et al., 1982) for offline analysis in MATLAB (The MathWorks).

We recorded the responses of a total of 226 neurons from cortical area MSTd in M606 and M125. Neurons with $<40$ trial repetitions of the delayed steer to Sample task (five trials/stimulus) were excluded from the analysis, leaving a total of 197 neurons. Responses to dot motion during the Sample or Start conditions were compared with activity with fixation alone (two-way ANOVA with/without dot motion, Sample/Start conditions). The great majority of MST neurons (172/197, 87.3\%) were dot motion or condition responsive and used in further analyses. There was no monkey $X$ stimulus interaction: neurons from both monkeys showed similar changes in cue selectivity across conditions.

Data analysis. Single-neuron studies presented a random sequence of heading stimuli during Sample and Start, with eight to 16 repetitions of each stimulus per condition. The monkeys were free move their eyes when steering, but all analysis periods were during centered fixation and stable joystick position. Responses in each condition were measured as firing rates in the last $200 \mathrm{~ms}$ of the Sample stimulus, the $200 \mathrm{~ms}$ before joystick rotation during the Start stimulus, and the $200 \mathrm{~ms}$ after the monkey completed joystick rotation during the Match condition. Selectivity for stimulus parameters was determined using a three-way ANOVA from responses to the three task conditions (Sample, Start, and Match), four COM locations (cardinal directions), and two radial motion patterns (in and out) in the MATLAB Statistics Toolbox. Population averages were determined by aligning responses to the preferred stimulus in Sample and normalizing to the mean firing rate to this stimulus.

Neuronal selectivity for the direction of local motion was characterized by calculating a local motion direction selectivity index defined as the contrast ratio of the average firing rate evoked by the preferred stimulus and the nonpreferred radial in/out pattern with the same COM location. Neuronal selectivity for the COM location was characterized by calculating a COM location selectivity index as the contrast ratio between the average firing rate evoked by the preferred stimulus and the nonpreferred radial in/out pattern at the antipreferred COM location. Both of these selectivity measures were calculated across the task using a $200 \mathrm{~ms}$ sliding window in $10 \mathrm{~ms}$ increments. 


\section{A Single Neuron Responses}
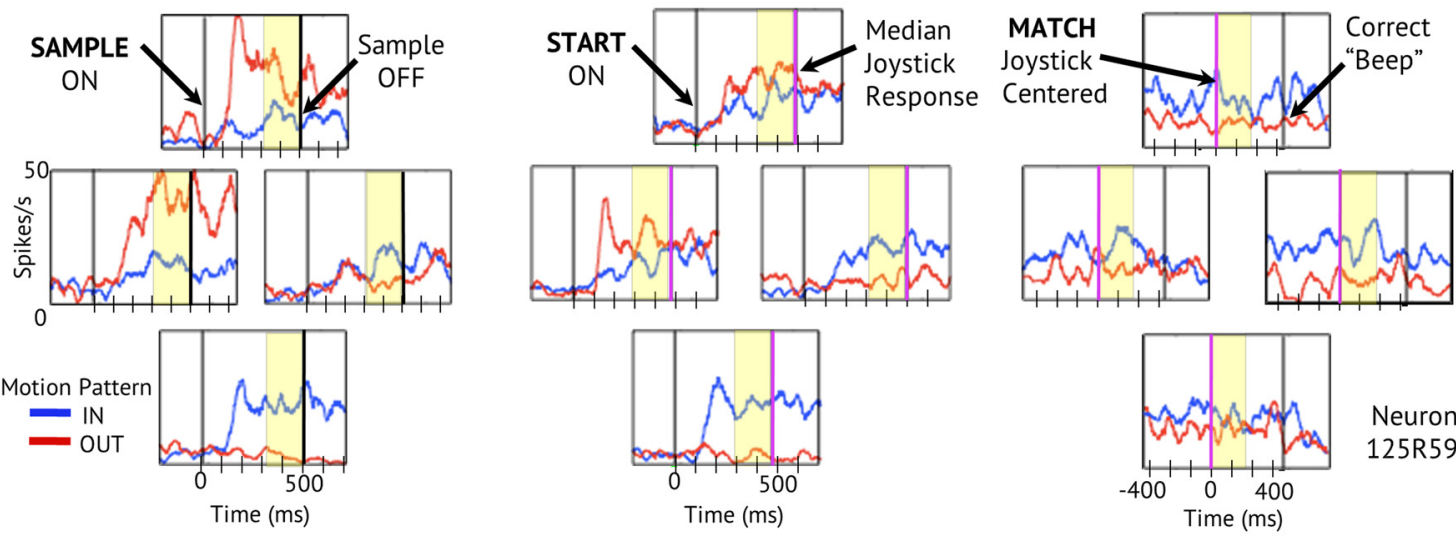

Neuron

B Selectivity Modulation

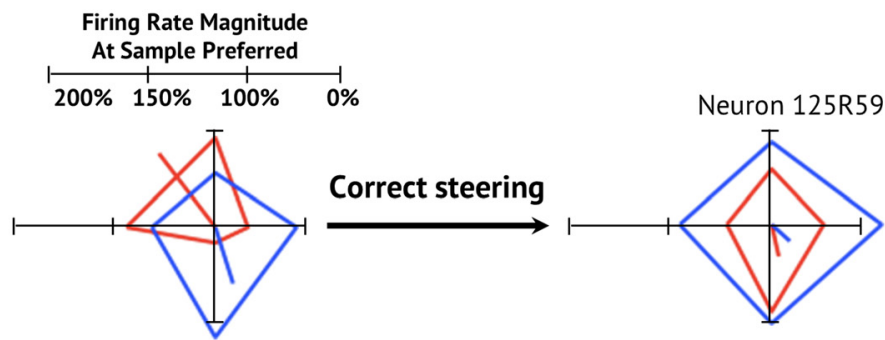

\section{Response Attenuation}
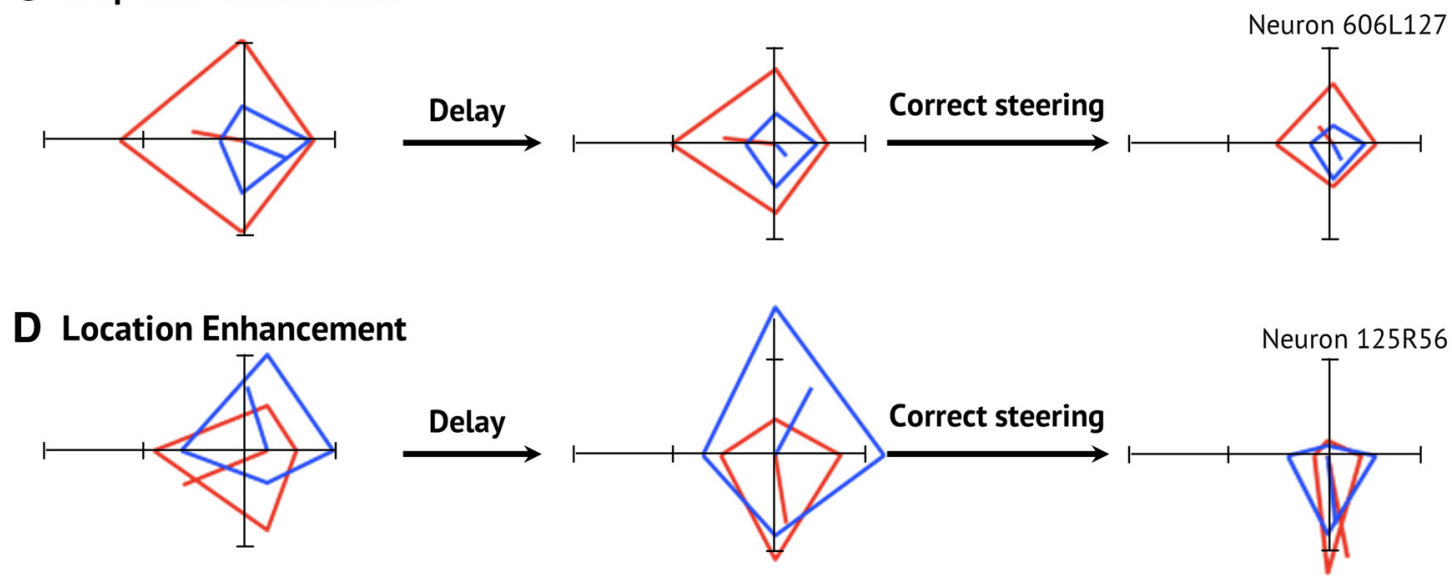

Figure 2. The diversity of single-neuron response changes across steering conditions. $A$, Neuron with robust motion pattern selectivity and modest heading location selectivity. Spike density functions (SDFs) are arranged at the four cardinal COM locations used during the Sample (left), Start (middle), and Match (right) conditions. Average responses to radial-in motion (blue) and radial-out motion (red) are shown for 16 correct trials for each optic flow stimulus (128 trials). The polar plots each group of SDFs represent average firing rate in the last $200 \mathrm{~ms}$ of each response period. The position of each apex corresponds to one of the four COM locations for inward and outward radial motion, with the co-plotted radial line representing the in and out net vectors. $\boldsymbol{B}$, This neuron shows decreased response selectivity from the Sample to the Start and Match conditions. C, Polar plots of the responses of a neuron that maintains selectivity but shows declining total response amplitude. C, Polar plots of the responses of a neuron that changes its stimulus selectivity to prefer inward and outward left side COMs. D, Polar plots of the responses of a neuron that demonstrates initial pattern selectivity changing to location selectivity during the match period.

\section{Results}

We recorded MSTd neuronal activity while monkeys were engaged in an active steering task in which imposed heading deviations must be rapidly corrected. Two monkeys were trained to view optic flow stimuli simulating heading directions distributed in heading space (Fig. 1B). These stimuli were presented in a behavioral task beginning with the monkeys holding a neutral steering wheel position while viewing a sample optic flow stimulus. That stimulus was followed by a second optic flow stimulus presenting a clockwise or counterclockwise heading deviation that signaled the monkey to start steering. The monkeys then turned a wheel to steer the optic flow back to match the original sample position (Fig. 1C). Both monkeys learned reliably accurate performance in the visual fixation and steering aspects of the tasks with minor idiosyncratic differences in the monkey's steering behavior (Fig. 1D).

We quantified these diverse task effects using a three-way ANOVA across neurons for task condition (Sample, Start, Match), radial pattern (in vs out), and COM location (four cardinal locations). The neuron shown in Figure 2, $A$ and $B$, changes its in versus out radial pattern selectivity and its preferred COM location from the Sample to the Match task conditions. It shows interaction effects between task condition $\times$ radial pattern $\left(F_{(2,405)}=4.40, p=0.013\right)$ 


\section{Population Average Responses}
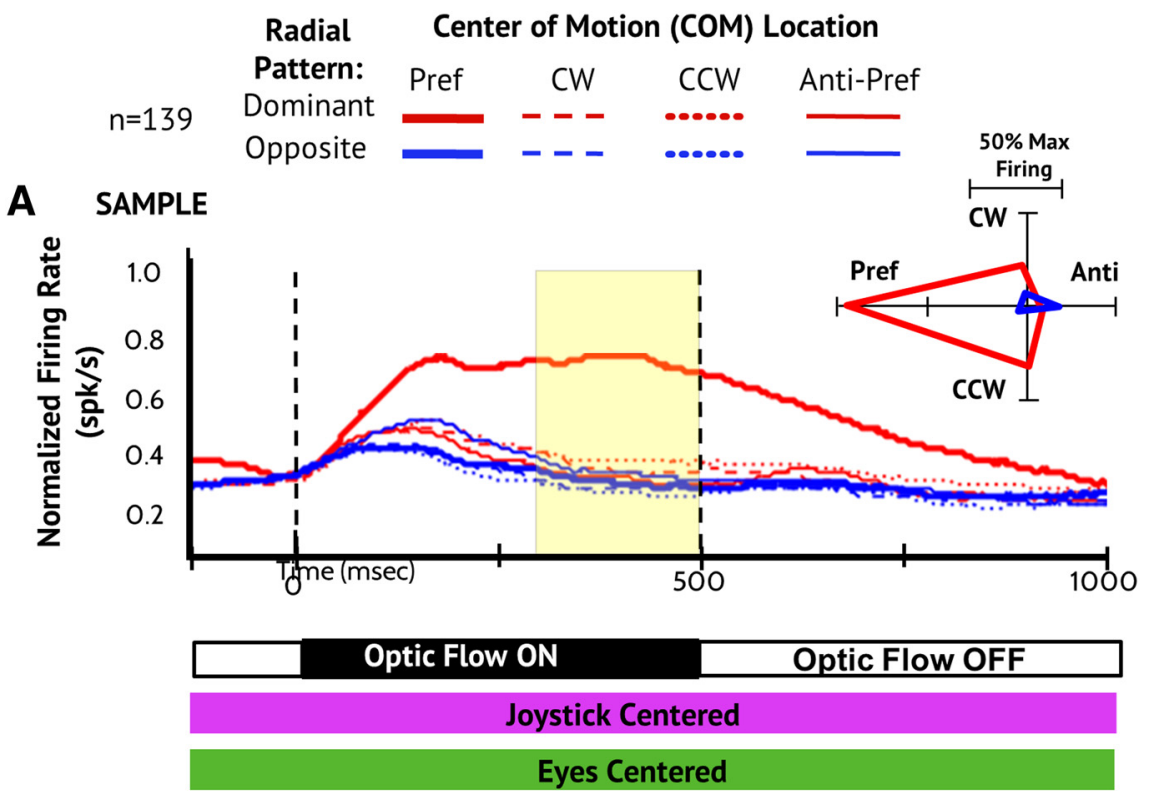

B
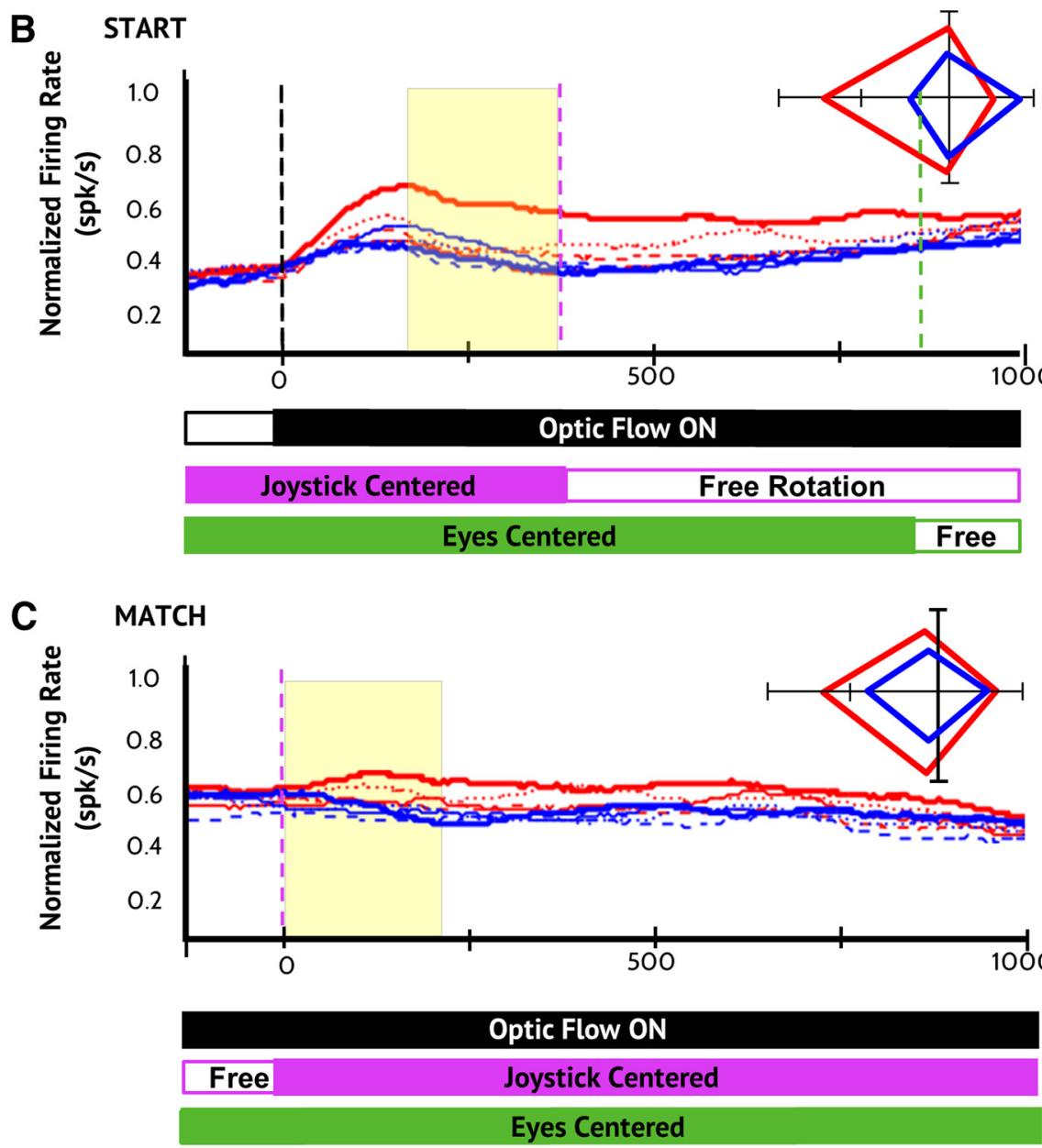

Figure 3. Population average responses for all neurons with significant optic flow selectivity during the steering task. $\boldsymbol{A}$, Averaged population neuronal responses aligned to the preferred optic flow stimulus in the Sample responses (thick solid red line) and normalized to the mean response rate evoked by that stimulus. Responses to the nondominant in/out radial motion pattern (blue), to the clockwise (CW; dashed), counterclockwise (CC; dotted), and anti-preferred (thin line) COM locations for the Sample $(\boldsymbol{A})$, Start $(\boldsymbol{B})$, and Match $(\boldsymbol{C})$ conditions. Polar plots (right) show the average firing rate at the four COM locations of the dominant (red) and nondominant (blue) radial patterns in the last $200 \mathrm{~ms}$ of the three conditions. Across the population, there and between task condition $\times$ radial pattern $\times$ COM location $\left(F_{(6,405)}=3.88, p<\right.$ $0.001)$.

In contrast, the neuron in Figure $2 C$ maintains its radial pattern and COM location selectivity, but shows decreasing net responsiveness across task conditions, which is reflected in a main effect of task condition $\left(F_{(2,600)}=39.4, p<0.001\right)$. Finally, the neuron in Figure $2 D$ changes both its preferred COM location and its net responsiveness, yielding a condition $\times$ location interaction effect $\left(F_{(6,180)}\right.$ $=2.34, p=0.034$ ).

The great majority of neurons yielded at least one significant effect in the condition $\times$ pattern $\times$ location ANOVAs $(74 \%, 139 / 188)$. These effects include main effects of task condition (57\%, 79/ $139)$, radial pattern $(42 \%, 58 / 139)$, and COM location $(40 \%, 55 / 131)$. In addition, there are a great many interaction effects across task conditions with task condition $\times$ radial pattern $(22 \%, 31 / 139)$, task condition $\times$ COM location $(33 \%, 46 /$ $139)$, and task condition $\times$ radial pattern $\times$ COM location $(28 \%, 39 / 139)$.

Despite the diversity of single neuron responses, the population averaged responses show distinctly different responses in the Sample, Start, and Match conditions. During the Sample period, the population response shows pronounced global pattern selectivity: the population responses to the preferred stimuli are larger than the responses to all other stimuli, those with the dominant radial pattern, and a great deal larger than those with the nondominant radial pattern (Fig. 3A).

Global pattern selectivity declines during the Start and remains low during the Match condition (Fig. 3B,C). We quantified this effect in a three-way ANOVA across neurons for task condition (Sample, Start, Match), radial pattern (in vs out), and COM location (four cardinal locations). This yields highly significant main effects of condition $\left(F_{(2,3312)}=\right.$ 13.46, $p<0.001)$.

Comparing the Sample and Start conditions reveals greatly increased responses

$\longleftarrow$

is a decrease in radial selectivity from Sample to Start and an increase in COM location selectivity from Start to Match. $\boldsymbol{B}$, Averaged neuronal population responses aligned to the preferred motion pattern in each of the response periods (thick solid red line) and normalized to the mean response rate evoked by that stimulus (format as in $\boldsymbol{A}$ ). Across the population, there are changes in which of the stimuli are preferred from Sample to Start and Match, with a subtle decline in selectivity (Sample to Start) and then a reversal of stimulus preferences to favor location effects (Start to Match). 


\section{Optic Flow Cue Selectivity}

A

\section{COM Location}

$$
\text { Index () }
$$
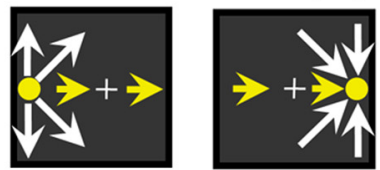

Preferred Opposite Location

n=139 Stimulus Same Direction

\section{COM Location Index}
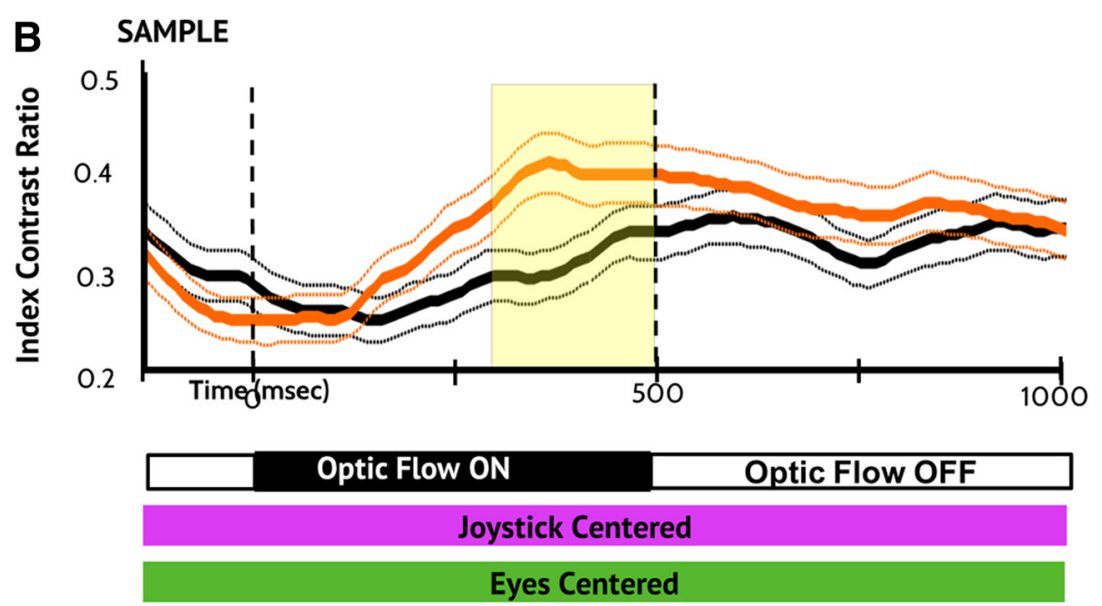

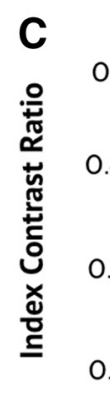

START

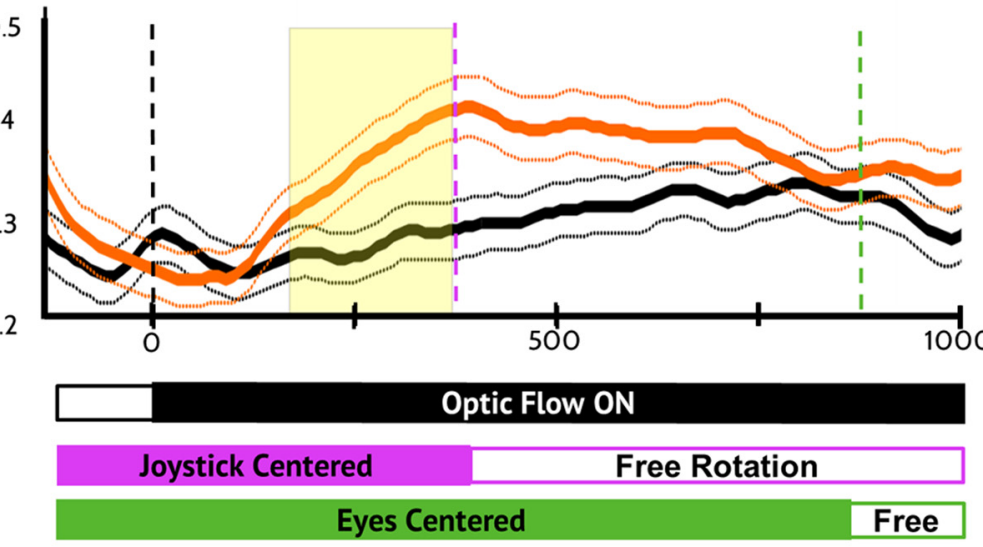

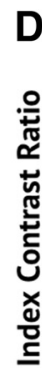

\section{MATCH}

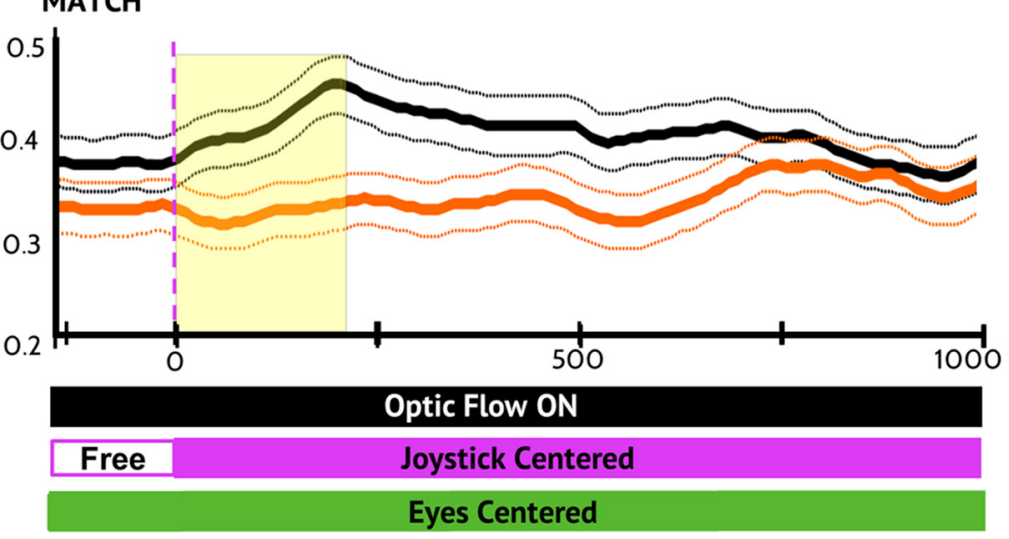

Figure 4. Population average selectivity indices across steering task conditions. $A$, The in/out radial pattern was randomized between Sample and Start to distinguish between the influence of local motion direction selectivity and COM location selectivity. to the nondominant in/out radial stimuli in Start (Fig. $3 A, B$ ). This yields significant interaction effects: condition $\times$ radial direction $F_{(2,2208)}=3.87, p=0.049$, Tukey HSD: nondominant radial during Sample $<$ during Start), and condition $X$ COM location $\left(F_{(3,2208)}=5.26, p=0.001\right.$; Tukey HSD: antipreferred COM during Sample $<$ during Start).

The loss of radial in/out selectivity could reflect our randomization of the radial pattern between Sample and Start, but that would not explain in/out having opposite COM location preferences. We examined this further by comparing trials in which Start and Sample stimuli were opposite radial patterns and trials in which they were the same. Only 8/139 neurons showed a significant $(p<0.05)$ main effect of a change in radial pattern from Sample to Start, suggesting that radial pattern changes do not underlie the change in optic flow preferences from Sample to Start.

The combined loss of radial-in versus radial-out selectivity, with opposite COM preferences, may be best explained as a shift from global radial pattern selectivity to local motion responsiveness. That is, a shift to responsiveness driven by a segment of the stimulus in which the direction of dot motion is the same for two optic flow stimuli having opposite radial directions and opposite COM locations. We have found previously that the dominant stimulus segment is in the central $20^{\circ}$ of the visual field, which is de-emphasized in responses focused on global pattern analysis and emphasized in responses focused on local motion analysis (Page and Duffy, 2003; Yu et al., 2010).

As in the Start condition, the Match responses show relatively little global pattern selectivity (Fig. 3C, bottom), with less responsiveness to the global pattern stimulus preferred in the Sample condition. A comparison between the Start and Match

\footnotetext{
The COM location selectivity index (yellow disk/icon) is the contrast ratio of response firing rates to the preferred stimulus and the stimulus with the opposite COM location and the same radial pattern (lefticons). The local motion direction selectivity index (yellow lines) is the contrast ratio of response firing rates to the preferred stimulus and the stimulus with the nondominant in/out radial motion and the same COM location (right icons). $\boldsymbol{B}-\boldsymbol{D}$, Time course of neuronal population selectivity for COM location and motion direction across the Sample $(\boldsymbol{B})$, Start (C), and Match (D) periods. During the Sample and Start periods, motion direction selectivity is more prominent than COM location selectivity. During the Match period, this relationship is reversed such that COM location selectivity is more prominent than local motion direction selectivity.
}

$\leftarrow$ 
conditions yields no main effect of condition or any condition $x$ stimulus interaction effects (all $p>0.60$ ).

We hypothesized that the loss of global pattern selectivity in the Start and Match conditions could reflect a shift in neuronal stimulus selectivity to different optic flow cues. This view was based on two contrived attributes of the paradigm: First, the task required the monkeys to steer the COM to match the Sample location. Second, the randomization of the in/out pattern would render global radial selectivity irrelevant. We tested this hypothesis by comparing the motion direction selectivity index versus the COM location selectivity index across task conditions (Fig. $4 A)$.

As the monkeys view the Sample optic flow to see the goal in each trial (Fig. 4B) and then view the Start optic flow to select the correct steering direction (Fig. 4C), the population maintains strong motion direction selectivity and weak COM location selectivity. In Sample, the neuronal population shows selective preferences of one of the eight optic flow stimuli. In Start, the population prefers in/out opposite COM stimuli. In Match, the neurons prefer same COM in/out stimuli. These observations are consistent with the view that MSTd neurons contribute to optic flow analysis using different response criteria, including shifting emphasis on global pattern, local motion, and COM location cues.

During the subsequent active steering period, the optic flow continually changes and both motion direction and COM location selectivity are reduced. Finally, when the monkey recenters the joystick and regains centered fixation, the optic flow comes to match the COM location in the Sample. At that point, population selectivity is the opposite of that seen in Sample and Start, with COM location selectivity exceeding motion direction selectivity (Fig. 4D). The marked change in the nature of optic flow selectivity is evidenced by a significant condition (Sample, Start, Match) $\times$ selectivity type (motion direction, COM location) interaction effect $\left(F_{(2,828)}=8.90, p<0.001\right)$.

Despite the uniformity of stimulus composition across steering conditions, MSTd neurons change their response properties to highlight the cue that is most relevant to the current task condition: in the Sample condition, global pattern selectivity of individual stimuli is at its greatest; in the Start condition, the representation of local motion directions increases; in the Match condition, the representation of COM location increases. Therefore, as changing steering conditions transform the task, changing cue preferences transform MSTd's optic flow responses.

\section{Discussion}

\section{Task-dependent stimulus-response relations}

Sample optic flow evokes highly selective neuronal population responses. This selectivity is consistent with the Sample's presenting one of eight, equally likely stimuli widely distributed in $3 \mathrm{D}$ heading space. The demand for the rapid and specific encoding of optic flow is further increased by the brevity of the Sample stimulus (500 ms) and its critical behavioral relevance in the task. Although the Sample evokes diverse single neuron responses (Fig. 2), aligning neuronal responses on their preferred heading directions and normalizing their response amplitudes creates a coherent representation of population responses (Georgopoulos et al., 1986; Ben Hamed et al., 2003).

These effects might reflect motion adaptation from sequential stimulation (Sekuler, 1965), particularly because Sample preferred stimuli evoke smaller responses in Start and Match (Fig. 3). Our paradigm always presented a series of orthogonal direction stimuli, not same or opposite directions, so classically described motion adaptation effects do not readily account for our obser- vations. Nevertheless, the selective adaptation of MSTd neuronal receptive field segments might reflect adaptation of input from adjacent MT (Boussaoud et al., 1990; VanWezel and Britten, 2002; Kohn and Movshon, 2004; Glasser et al., 2011). However, the distinct effects seen from Sample to Start and from Start to Match are unlikely to be from the same, single underlying response mechanism.

Our findings substantially alter earlier conclusions about task effects in MSTd. Comparing optic flow used to guide saccades with optic flow that is behaviorally irrelevant shows that relevance enhances MSTd's response amplitude and heading selectivity (Treue and Maunsell, 1996; Dubin and Duffy, 2007). When optic flow guides button presses in a delayed match to Sample task, stronger selectivity is evoked by the Sample than by the match or nonmatch (Sato et al., 2013). The current study confirms that behaviorally relevant Sample stimuli evoke strong heading selectivity and also shows that the fundamental stimulus-response relations of single neurons can be changed by ongoing behavior.

\section{Mechanisms of stimulus selectivity}

MSTd's optic flow responses change from Sample to Start, with larger Start responses to the Sample's nonpreferred in/out radial direction (Fig. 3B). Critically, in/out radial patterns sharing the same local motion directions at locations around the central visual field have opposite COM locations. This allows us to distinguish local motion and COM location effects. It also suggests that the shift to local motion responses may reflect changes in the responsiveness of receptive field segments that are selectively activated by a particular direction of planar motion.

An alternative view might attribute response differences among Sample, Start, and Match from the stimulus sequence independent of the task in which it is presented. From this perspective, the Sample stimulus can be seen as being presented on an unbiased clean slate of neuronal responsiveness. The Start stimulus is presented in the context of the preceding Sample stimulus; the Match stimulus in the context of the Sample, Start, and Steering period stimuli. The visual context of these stimuli may contribute to the observed response changes. However, previous studies of MSTd neuronal responses to series of optic flow stimuli have not revealed these effects (Paolini et al., 2000; Froehler and Duffy, 2002; Page et al., 2015). We suggest that the naturalistic behavioral task that links the Sample, Start, and Match conditions may influence neuronal responses changes across those conditions. Further, we expect that the varying behavioral significance of cues imbedded in optic flow may be reflected in the neuronal responses to optic flow in each task condition.

A neural network model of MST responses demonstrated that responses to radial-in and radial-out optic flow stimuli with opposite COM locations can emerge from the input of MT-like neurons (Lappe et al., 1996). This suggests that the steering effects that we have seen in MSTd are consistent with underlying planar motion mechanisms. More recent studies suggest that the response dynamics revealed by the network elements may reflect direction-specific interactions between receptive field segments that could be driven by input from MT (Khawaja et al., 2009; Yu et al., 2010).

The current findings further suggest that task effects can shift MSTd neurons from their intrinsic optic flow selectivity to more planar motion dependent, MT-like responses. This occurs despite our promoting global pattern analysis by randomizing radial in/out directions between Sample and Start (O'Brien et al., 2001; Page and Duffy, 2008). We consider that the Sample COM may focus the monkey on that segment of the visual field and enhance responses to planar motion at that site in a transition of 
spatial attention from a floodlight to a spotlight (Posner et al., 1980) or from attention to the stimulus to intention in the task (Snyder et al., 2000).

\section{Highlighting the destination}

The Match condition yields greater influence of COM location, seen as similar responsiveness to radial in/out directions with the same spatial location of the COM, be it a center of expansion from outward radial movement or a center of contraction from inward radial movement (Fig. 3C). These effects are seen in comparisons of motion and location selectivity across task conditions, with stronger motion selectivity in Sample and Start and stronger COM location selectivity in Match (Fig. 4). The influence of COM location in Match responses is consistent with the behavioral goal of the Match condition: to steer the optic flow's COM location to match that presented in the Sample.

Optic flow provides a wide variety of cues about the observer's self-movement heading direction. These include the global radial motion pattern, the local planar motion at different points in the visual field, and the spatial location of the radial COM. There are still more visual cues in optic flow that are not explicitly consider in this analysis, such as speed gradients, texture and occlusion depth cues, and derived or extracted motion parameters (Gibson, 1966; Dosher et al., 1989; Landy et al., 1991; Cornilleau-Pérès and Gielen, 1996; Calow and Lappe, 2007). Our result suggests that when the goal of ongoing behavior is to detect a change in the flow field, the neuronal population transforms to better represent local motion directions. When the goal of ongoing behavior is to monitor the spatial location of the COM, the neuronal population transforms to better represent spatial location.

Steering-related changes in neuronal optic flow preferences may be similar to featural attentional effects on natural scene preferences, with neuronal (David et al., 2008) and hemodynamic (Çukur et al., 2013) changes related to task demands. Such effects may be mediated by top-down connections from prefrontal to posterior cortical areas (Buschman and Miller, 2007) transmitting signals that reflect the dynamic encoding of behavioral tasks in prefrontal cortex (Stokes et al., 2013).

Alternatively, stimulus preference changes in MSTd might not be explicitly controlled, but rather may reflect MSTd's involvement in a distributed representation of the learned steering task such that the monkey anticipates the next task condition and intends a behavioral response to that condition (Lashley, 1929; Mountcastle and Edelman, 1978). Changes across the sensorymotor stages of the steering task may trigger the contextual reoptimization of cortical neuronal assemblies without explicit control signals (Singer, 2009; Friston, 2010), potentially by reweighting local motion input from MT and spatial location signals from PPC. Therefore, steering could transform MSTd's optic flow responses from its intrinsic global pattern analysis to MTlike local motion analysis and then to PPC-like spatial location analysis.

\section{References}

Andersen RA, Asanuma C, Essick G, Siegel RM (1990) Corticocortical connections of anatomically and physiologically defined subdivisions within the inferior parietal lobule. J Comp Neurol 296:65-113. CrossRef Medline

Ben Hamed S, Page W, Duffy C, Pouget A (2003) MSTd neuronal basis functions for the population encoding of heading direction. J Neurophysiol 90:549-558. CrossRef Medline

Bisley JW, Goldberg ME (2003) Neuronal activity in the lateral intraparietal area and spatial attention. [see comment]. Science 299:81-86. CrossRef Medline
Boussaoud D, Ungerleider LG, Desimone R (1990) Pathways for motion analysis: cortical connections of the medial superior temporal and fundus of the superior temporal visual areas in the macaque. J Comp Neurol 296:462-495. CrossRef Medline

Buschman TJ, Miller EK (2007) Top-down versus bottom-up control of attention in the prefrontal and posterior parietal cortices. Science 315: 1860-1862. CrossRef Medline

Britten KH, Van Wezel RJ (2002) Area MST and heading perception in macaque monkeys. Cereb Cortex 12:692-701. CrossRef Medline

Calow D, Lappe M (2007) Local statistics of retinal optic flow for selfmotion through natural sceneries. Network 18:343-374. CrossRef Medline

Cornilleau-Pérès V, Gielen CC (1996) Interactions between self-motion and depth perception in the processing of optic flow. Trends Neurosci 19:196-202. CrossRef Medline

Crist CF, Yamasaki DS, Komatsu H, Wurtz RH (1988) A grid system and a microsyringe for single cell recordings. J Neurosci Methods 26:117-122. CrossRef Medline

Çukur T, Nishimoto S, Huth AG, Gallant JL (2013) Attention during natural vision warps semantic representation across the human brain. Nat Neurosci 16:763-770. CrossRef Medline

David SV, Hayden BY, Mazer JA, Gallant JL (2008) Attention to stimulus features shifts spectral tuning of V4 neurons during natural vision. Neuron 59:509-521. CrossRef Medline

Dosher BA, Landy MS, Sperling G (1989) Kinetic depth effect and optic flow. I. 3D shape from Fourier motion. Vision Res 29:1789-1813. CrossRef Medline

Dubin MJ, Duffy CJ (2007) Behavioral influences on cortical neuronal responses to optic flow. Cereb Cortex 17:1722-1732. CrossRef Medline

Duffy CJ, Wurtz RH (1991a) Sensitivity of MST neurons to optic flow stimuli. I. A continuum of response selectivity to large-field stimuli. J Neurophysiol 65:1329-1345. Medline

Duffy CJ, Wurtz RH (1991b) Sensitivity of MST neurons to optic flow stimuli. II. Mechanisms of response selectivity revealed by small-field stimuli. J Neurophysiol 65:1346-1359. Medline

Duffy CJ, Wurtz RH (1995) Response of monkey MST neurons to optic flow stimuli with shifted centers of motion. J Neurosci 15:5192-5208. Medline

Friston K (2010) The free-energy principle: a unified brain theory? Nat Rev Neurosci 11:127-138. CrossRef Medline

Froehler MT, Duffy CJ (2002) Cortical neurons encoding path and place: where you go is where you are. Science 295:2462-2465. CrossRef Medline

Georgopoulos AP, Schwartz AB, Kettner RE (1986) Neuronal population coding of movement direction. Science 233:1416-1419. CrossRef Medline

Gibson JJ (1950) The perception of the visual world. Boston: Houghton Mifflin.

Gibson JJ (1966) The senses considered as perceptual systems. Boston: Houghton Mifflin.

Glasser DM, Tsui JM, Pack CC, Tadin D (2011) Perceptual and neural consequences of rapid motion adaptation. Proc Natl Acad Sci U S A 108: E1080-E1088. CrossRef Medline

Hays AV, Richmond BJ, Optican LM (1982) A UNIX-based multiple process system for real-time data acquisition and control. WESCON Conference Proceedings 2:1-10.

Herrington TM, Assad JA (2010) Temporal sequence of attentional modulation in the lateral intraparietal area and middle temporal area during rapid covert shifts of attention. J Neurosci 30:3287-3296. CrossRef Medline

Judge SJ, Richmond BJ, Chu FC (1980) Implantation of magnetic search coils for measurement of eye position: an improved method. Vision Res 20:535-538. CrossRef Medline

Khawaja FA, Tsui JM, Pack CC (2009) Pattern motion selectivity of spiking outputs and local field potentials in macaque visual cortex. J Neurosci 29:13702-13709. CrossRef Medline

Kishore S, Hornick N, Sato N, Page WK, Duffy CJ (2012) Driving strategy alters neuronal responses to self-movement: cortical mechanisms of distracted driving. Cereb Cortex 22:201-208. CrossRef Medline

Kohn A, Movshon JA (2004) Adaptation changes the direction tuning of macaque MT neurons. Nat Neurosci 7:764-772. CrossRef Medline

Komatsu H, Wurtz RH (1988) Relation of cortical areas MT and MST to pursuit eye movements. I. Localization and visual properties of neurons. J Neurophysiol 60:580-603. Medline 
Landy MS, Dosher BA, Sperling G, Perkins ME (1991) The kinetic depth effect and optic flow. II. First- and second-order motion. Vision Res 31:859-876. CrossRef Medline

Lappe M, Bremmer F, Pekel M, Thiele A, Hoffmann KP (1996) Optic flow processing in monkey STS: a theoretical and experimental approach. J Neurosci 16:6265-6285. Medline

Lashley KS (1929) Brain mechanisms and intelligence, pp 186. Chicago: Chicago UP.

Mountcastle VB, Edelman GM (1978) The mindful brain: cortical organization and the group-selective theory of higher brain function. Cambridge, MA: MIT.

O’Brien HL, Tetewsky SJ, Avery LM, Cushman LA, Makous W, Duffy CJ (2001) Visual mechanisms of spatial disorientation in Alzheimer's disease. Cereb Cortex 11:1083-1092. CrossRef Medline

Page WK, Duffy CJ (2003) Heading representation in MST: sensory interactions and population encoding. J Neurophysiol 89:1994-2013. Medline

Page WK, Duffy CJ (2008) Cortical neuronal responses to optic flow are shaped by visual strategies for steering. Cereb Cortex 18:727-739. CrossRef Medline

Page WK, Sato N, Froehler MT, Vaughn W, Duffy CJ (2015) Navigational path integration by cortical neurons: origins in higher-order direction selectivity. J Neurophysiol 113:1896-1906. CrossRef Medline

Paolini M, Distler C, Bremmer F, Lappe M, Hoffmann KP (2000) Responses to continuously changing optic flow in area MST. J Neurophysiol 84: 730-743. Medline

Posner MI, Snyder CR, Davidson BJ (1980) Attention and the detection of signals. J Exp Psychol 109:160-174. CrossRef Medline

Saalmann YB, Pigarev IN, Vidyasagar TR (2007) Neural mechanisms of visual attention: how top-down feedback highlights relevant locations. Science 316:1612-1615. CrossRef Medline

Sato N, Page WK, Duffy CJ (2013) Task contingencies and perceptual strategies shape behavioral effects on neuronal response profiles. J Neurophysiol 109:546-556. CrossRef Medline

Seidemann E, Newsome WT (1999) Effect of spatial attention on the responses of area MT neurons. J Neurophysiol 81:1783-1794. Medline
Sekuler RW (1965) Spatial and temporal determinants of visual backward masking. J Exp Psychol 70:401-406. CrossRef Medline

Singer W (2009) The brain, a complex self-organizing system. European Review 17:321-329. CrossRef

Snyder LH, Batista AP, Andersen RA (2000) Intention-related activity in the posterior parietal cortex: a review. Vision Res 40:1433-1441. CrossRef Medline

Stokes MG, Kusunoki M, Sigala N, Nili H, Gaffan D, Duncan J (2013) Dynamic coding for cognitive control in prefrontal cortex. Neuron 78: 364-375. CrossRef Medline

Tanaka K, Saito H (1989) Analysis of motion of the visual field by direction, expansion/contraction, and rotation cells clustered in the dorsal part of the medial superior temporal area of the macaque monkey. J Neurophysiol 62:626-641. Medline

Treue S, Martínez Trujillo JC (1999) Feature-based attention influences motion processing gain in macaque visual cortex. Nature 399:575-579. CrossRef Medline

Treue S, Maunsell JH (1996) Attentional modulation of visual motion processing in cortical areas MT and MST. Nature 382:539-541. CrossRef Medline

Ungerleider LG, Mishkin M (1982) Two cortical visual systems. In: Analysis of visual behavior (Ingle DJ, Goodale MA, Mansfield RJW, eds), pp 549-586. Cambridge: MIT.

Van Wezel RJ, Britten KH (2002) Motion adaptation in area MT. J Neurophysiol 88:3469-3476. CrossRef Medline

Warren WH (1995) Self-motion: visual perception and visual control. in: perception of space and motion, pp 263-325. San Diego: Academic.

Wegener D, Freiwald WA, Kreiter AK (2004) The influence of sustained selective attention on stimulus selectivity in macaque visual area MT. J Neurosci 24:6106-6114. CrossRef Medline

Yu CP, Page WK, Gaborski R, Duffy CJ (2010a) Receptive field dynamics underlying MST neuronal optic flow selectivity. J Neurophysiol 103: 2794-2807. CrossRef Medline

Zeki SM (1974) Functional organization of a visual area in the posterior bank of the superior temporal sulcus of the rhesus monkey. J Physiol 236:549-573. CrossRef Medline 